\title{
Expected gain in the pyramid wavefront sensor with limited Strehl ratio
}

\author{
V. Viotto, R. Ragazzoni, M. Bergomi, D. Magrin, and J. Farinato
}

\begin{abstract}
INAF-Osservatorio Astronomico di Padova, Vicolo dell'Osservatorio 5, 35122 Padova, Italy e-mail: valentina.viotto@oapd.inaf.it
\end{abstract}

Received 21 December 2015 / Accepted 30 June 2016

\begin{abstract}
Context. One of the main properties of the pyramid wavefront sensor is that, once the loop is closed, and as the reference star image shrinks on the pyramid pin, the wavefront estimation signal-to-noise ratio can considerably improve. This has been shown to translate into a gain in limiting magnitude when compared with the Shack-Hartmann wavefront sensor, in which the sampling on the wavefront is performed before the light is split into four quadrants, which does not allow the quality of the focused spot to increase. Since this property is strictly related to the size of the re-imaged spot on the pyramid pin, the better the wavefront correction, the higher the gain. Aims. The goal of this paper is to extend the descriptive and analytical computation of this gain that was given in a previous paper, to partial wavefront correction conditions, which are representative for most of the wide field correction adaptive optics systems.

Methods. After focusing on the low Strehl ratio regime, we analyze the minimum spatial sampling required for the wavefront sensor correction to still experience a considerable gain in sensitivity between the pyramid and the Shack-Hartmann wavefront sensors. Results. We find that the gain can be described as a function of the sampling in terms of the Fried parameter.
\end{abstract}

Key words. instrumentation: adaptive optics

\section{Introduction}

It has been almost twenty years since the pyramid wavefront sensor (P-WFS) was first proposed (Ragazzoni 1996) as an alternative to other types of wavefront derivative measuring sensors. The first single star adaptive optics (AO) loop with a P-WFS was closed on AdOpt@TNG (Ragazzoni et al. 2000a), and one of the P-WFS best results on sky came recently with FLAO (Esposito et al. 2011), the First Light Adaptive Optics system at the $8 \mathrm{~m}$ Large Binocular Telescope (LBT; Hill \& Salinari 2000), which further increased the popularity of the pyramid sensor. This kind of wavefront sensor has also been successfully implemented in the configuration of multi conjugated adaptive optics (MCAO; Beckers 1988), during the MCAO demonstrator (MAD) experiment at the Very Large Telescope (Marchetti et al. 2007; Arcidiacono et al. 2004) and it is the heart of the next Fizeau interferometric MCAO-equipped focal station LINCNIRVANA (Herbst et al. 2003) for the LBT, which couples the layer-oriented technique (Ragazzoni et al. 2000b) with the multiple field of view concept (Ragazzoni et al. 2002). Moreover, the P-WFS has been studied and implemented in other science fields, like ophthalmology (Iglesias et al. 2002; Chamot et al. 2006) or phase microscopy (Iglesias 2011). Nowadays, the P-WFS is considered a very powerful and promising tool both for extreme $\mathrm{AO}$ and for MCAO for wide corrected fields of view (Kasper et al. 2014). Being the core of very different systems and concepts, it is not, however, always used in the same configuration, so its properties are exploited in different ways. While, in the following, we examine the difference in performance between the P-WFS and the Shack-Hartmann wavefront sensor (SH-WFS), it is noticeable that these do not exhaust the whole range of WFSs available in AO and specifically for astronomical purposes. Adopting the distinction between WFSs whose detection is performed in the pupil rather than the object plane, we would like to mention, in the first group, the curvature (Roddier 1981) wavefront sensor. This is, however, sensitive to the second derivative (Laplacian) of the wavefront rather than the first derivative, a common property of P-WFS and SH-WFS. Various kinds of shearing interferometers (Hardy et al. 1977) have been implemented as well. Another interesting WFS is represented by the Smartt (Smartt \& Strong 1974) in which a signal almost proportional to the wavefront departure from flatness is available, when used close to the diffraction limit condition.

\section{Approach and assumptions}

In Ragazzoni \& Farinato (1999), the authors investigated the non-modulated P-WFS sensitivity with respect to an SH-WFS in perfect closed loop conditions and carried out an analysis on the gain of the P-WFS in terms of guide star limiting magnitude, with respect to the SH-WFS. (In this paper, we will refer at this parameter as $\Delta_{\mathrm{MAG}}$.) The result comes from the fact that the variance of the P-WFS measurements is the same as the SHWFS for the highest sampled spatial frequency, but it decreases to a factor $\left(D / r_{0}\right)^{2}$ times lower than the SH-WFS for low order modes. This applies to the P-WFS when it is pushed to its best regime, i.e. when the loop is successfully closed with the highest number of modes allowed by a pupil sampling on scales of the order of $r_{0}$. The predicted gain was confirmed shortly after using numerical simulations (Esposito \& Riccardi 2001) and on-sky measurements (Ghedina et al. 2003). The sensitivity of the two mentioned WFSs has also been compared in the case of a modulating pyramid in Verinaud (2004), while a more extended comparison, which includes also other WFS techniques, such as the curvature WFS (Roddier \& Roddier 1988), has been 
presented by Guyon (2010). In this paper, we use the same approach and arguments adopted by Ragazzoni \& Farinato in the mentioned paper, but consider the possibility which happens, for example in MCAO or in open loop configuration, that the pyramid is not working under perfect illumination conditions, meaning that the correction of the wavefront is only partial. The computations and results are mostly focused on the pupil size regime of the next generation of large telescopes, such as the European Extremely Large Telescope (Gilmozzi \& Spyromilio 2007), the Giant Magellan Telescope (Johns 2008), and the Thirty Meter Telescope (Szeto et al. 2008), generically referred to as extremely large telescopes (ELTs). Here we intend to consider a P-WFS and an SH-WFS, working with the same main parameters, with the goal of obtaining a diffraction limited correction. In particular, unless differently specified and independent from the kind of WFS considered, in this paper we always assume the pupil as being sampled at $r_{0}$ scale. Moreover, the following discussion, analytical computations, and simulations only apply to AO systems using unresolved sources. Extended references that can be resolved by the combination of WFS and telescope, such as asteroids (Ribak \& Rigaut 1994), extragalactic nuclei (Le Louarn et al. 1998), laser guide stars (Foy \& Labeyrie 1985), and even, for ELTs, some natural guide stars (Weiner et al. 2000), are not treated here. For the variety of cases that we consider in this work, in open loop, the spots focusing on the wavefront derivative sensitive element of the two systems (i.e. the pyramid pin, for the P-WFS, and the detector, for the SH-WFS) share approximately the same size, which is related to the Fried parameter of the atmosphere. Once the loop is closed, however, in a geometrical approximation, the correction performed on the wavefront has no impact on the SH-WFS performance, while it allows the spot on the pyramid pin to shrink by a factor of $\sim D / r_{0}$, increasing the sensor signal-to-noise ratio (S/N). In Ragazzoni \& Farinato (1999), the authors analytically compute the effect of this spot-shrinking on the error that is associated with the tip-tilt measurement. Then they use the Heisenberg uncertainty principle to extend the same argument to the error of the P-WFS, with respect to the SH-WFS variance, for a Zernike polynomial of $q$ th radial order. While the same conclusions can be attained through Fourier optics, the Heisenberg principle is applied to the combined uncertainties on the position over the pupil and the momentum associated with the incoming direction, along an axis lying on the pupil plane. Diffraction limited performances are classically obtained through the same argument. The resulting dependence of the measurement variance on the considered radial order is

$\sigma_{P-Z(q)}^{2} \sim\left(\frac{q}{Q}\right)^{2} \sigma_{\mathrm{SH}-Z(q)}^{2}$,

where $q$ is the Zernike radial order and $Q=D / r_{0}$ is the maximum radial order that can be corrected by a system with an aperture $D$ (assuming sampling down to $r_{0}$ scale), when $r_{0}$ is the Fried parameter. To quantify the actual noise for the SH wavefront sensor, the result given in Rigaut \& Gendron (1992) was considered as a gauging point. They derived a general expression for the noise propagation coefficients of each Zernike mode in the case of an SH-WFS, to be applied to the noise which affects the slope measurement of each sub-aperture. This result, in which spatial and temporal errors are neglected, leads to a global reconstruction noise given by

$\sigma_{\mathrm{SH}}^{2}=\sigma_{\mathrm{ph}}^{2} \sum_{q=1}^{Q}\left[0.590(q+1)^{-2.05}+0.174(q-1)(q+1)^{-2}\right]$,

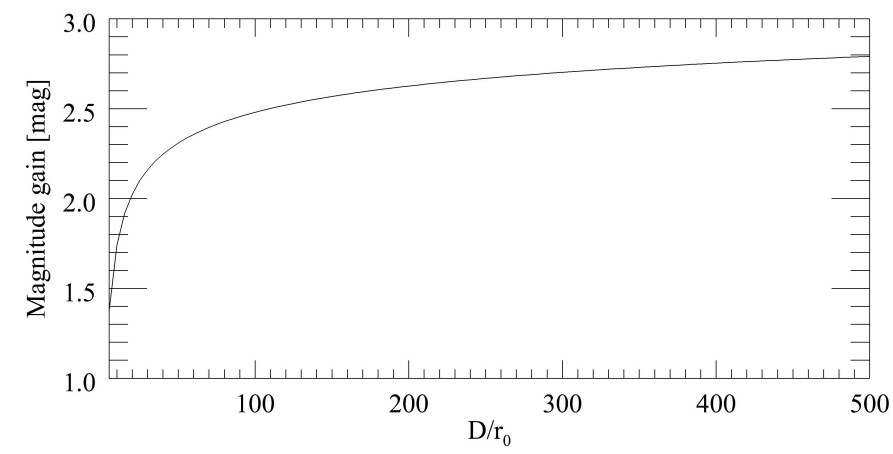

Fig. 1. P-WFS vs. SH-WFS magnitude gain at different maximum radial orders. Data taken from Ragazzoni \& Farinato (1999).

where $\sigma_{\mathrm{ph}}^{2}$ is the photonic noise error. Even if this analytical expression is not directly used in the following, here it is reported to show how the SH-WFS global reconstruction noise is proportional to the photon noise, enabling us to gauge the P-WFS noise to that of the SH-WFS, assuming the same reconstructor. The prediction given in 1999 was confirmed with high confidence for low radial orders (up to $Q=7$ ) with a laboratory experiment called Pyramir (Peter et al. 2010). We contacted the authors and were informed that their data were obtained in a laboratory and that the Strehl ratio (SR) on the pyramid pin was of the order of 90-95\% (priv. comm., 2012). From a course inspection of Fig. 8 of Peter et al. (2010), a level of confidence better than 5\% is estimated. The lower propagation coefficients, for the P-WFS in closed loop, translate into a gain $\Delta_{\mathrm{MAG}}>2.5 \mathrm{mag}$, which can be achieved with respect to a SH-WFS, for the generation of ELTs in the visible and IR bands (Fig. 1, reconstructed from the results in Ragazzoni \& Farinato 1999). A few words are required to explain the expression "gain in magnitude". In the framework of this paper, this type of gain is computed using the ratio of the noise coefficients for the P-WFS vs. conventional wavefront sensing approach. This gain will only affect the area of the plot SR vs. magnitude, where the SR is not saturated because of the dominance of other errors, especially the fitting one. In the intermediate and low SR regime, under this type of assumption, a curve that is rigidly shifted towards higher magnitudes should be experienced. It is clear, however, that for extremely low SRs, whose limit is dictated by the proximity to the minimum SR defined in the next section, the assumptions behind the calculation are not confirmed. For completeness, we must say that a similar result has been differently quantified by Verinaud (2004) with another model, which includes the filtering effect of the subapertures, resulting in a maximum $\Delta_{\mathrm{MAG}}$ that is smaller by about 0.5 mag than the one shown in Fig. 1 .

\section{Moving away from the high SR regime}

\subsection{Strehl ratio effect on tip-tilt determination error}

The overall gain in magnitude that results from this prediction can approximate the actual P-WFS $\Delta_{\text {MAG }}$ only assuming noiseless measurements or, in other words (at least in this context), considering the full correction achievable in the bright-end regime. To estimate the effect of a partial correction onto the pyramid pin (in the diffraction-to-seeing limit regime), we introduce the approximation that the shape of the re-imaged star is obtained superposing

- a seeing-limited spot, with a total flux of $(1-S) \times n^{*}$ photons - a diffraction-limited spot, produced by $S \times n^{*}$ photons, 


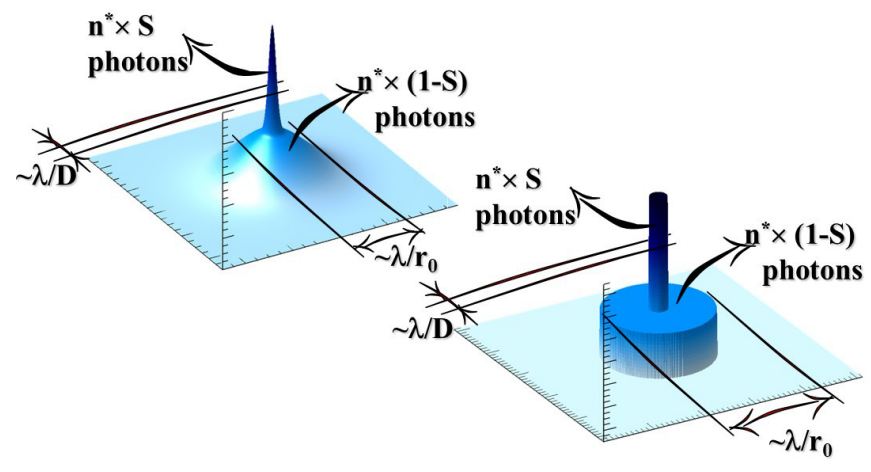

Fig. 2. Partial correction spot shape assumption. Top-left: Gaussian spot, bottom-right: cylindrical spot (scales are different).

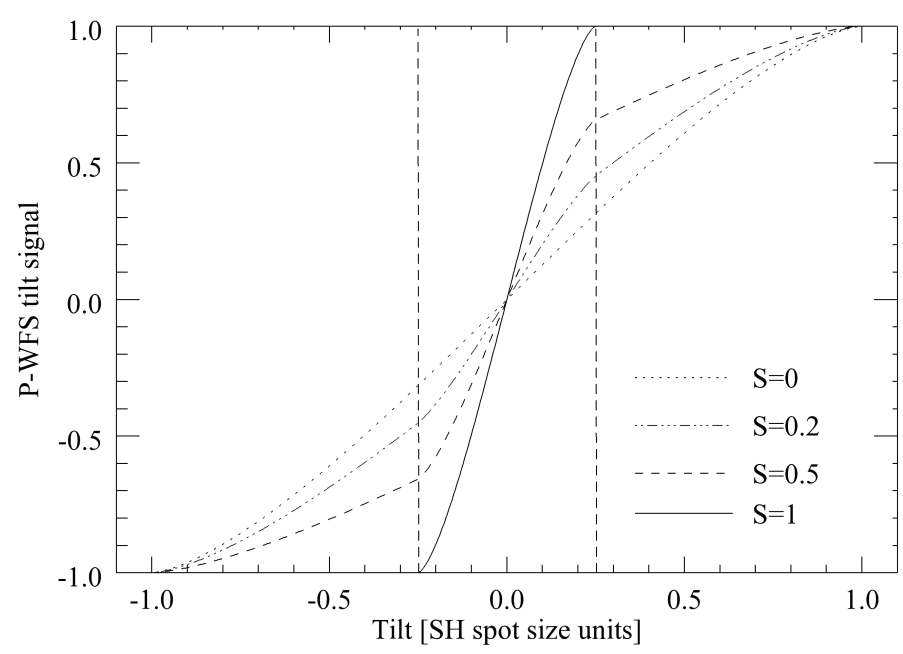

Fig. 3. P-WFS tilt signal slope in case of different SR achieved on the pyramid pin (a low value for $Q=D / r 0=4$ has been used for the purpose of clarity).

where $n^{*}$ is the total number of collected photons and $S$ the SR on the pyramid pin. On first analysis, the two superposed spots have been approximated with cylinders (Fig. 2). The definitions above implicitly assume that the central core of the PSF is clearly distinguishable from its surrounding halo. One can estimate the minimum SR, for which this assumption is verified: since the PSF comprises $N_{\mathrm{s}} \sim\left(D / r_{0}\right)^{2}$ speckles, a central peak one order of magnitude brighter than the halo could be obtained only for $\mathrm{SR}>S_{\min }=10 / N_{\mathrm{s}}$. While, for an ELT-like telescope, this is a very weak constraint, which leads to numbers usually well below $S_{\min }=1 \%$; for $8 \mathrm{~m}$-class telescopes a SR of the order of $10 \%$ is instead required.

Figure 3 shows the P-WFS tip-tilt signal response, as a function of the actual shift of the spot, for different values of the SR. We recall here that, for this kind of WFS, a steeper slope in the signal vs. shift plane describes a higher sensitivity. The latter can be reached either when a higher SR is delivered to the P-WFS, or, for a given $\mathrm{SR}$, when the $D / r_{0}$ parameter increases. In this last case, the signal response experiences a reduction of its linear range, which is included between the vertical dashed lines in Fig. 3.

With the afore-mentioned assumptions, we can derive analytically the dependence of the P-WFS tip-tilt error on the SR

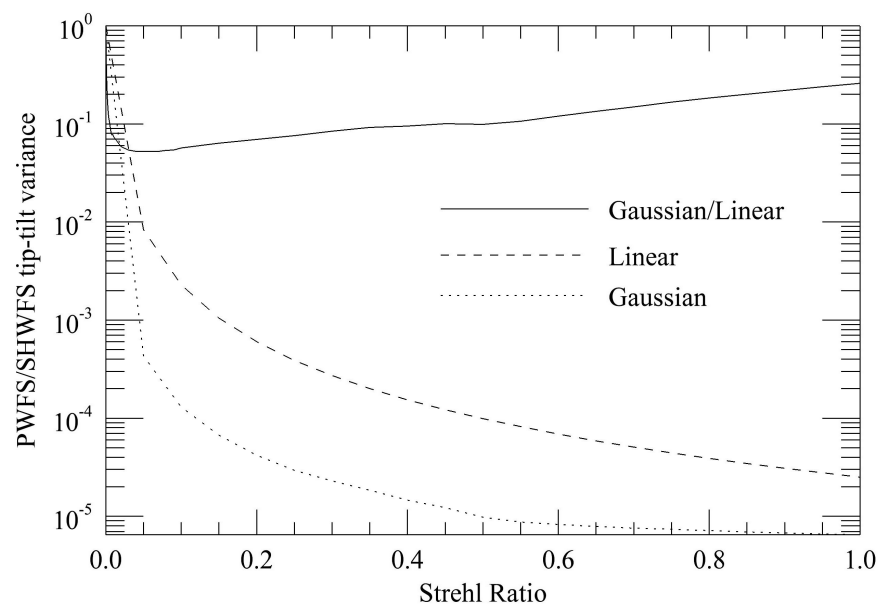

Fig. 4. Correction to be applied (solid line) if Gaussian-shaped spots (dotted line) are considered instead of cylindrical spots (dashed line). An E-ELT-like telescope in $r_{0}=0.2 \mathrm{~m}$ conditions has been assumed $\left(D / r_{0} \approx 200\right)$.

value, for small tip-tilt signals, as

$\sigma_{P-\mathrm{TT}}^{2} \approx\left[S \cdot\left(\frac{D}{r_{0}}-1\right)+1\right]^{-2} \sigma_{\mathrm{SH}-\mathrm{TT}}^{2}$.

For the sake of completeness, we add a further consideration: the cylindrical spots approximation clearly does not reproduce the actual shape of the spot on the pyramid pin, but it tends to underestimate the sensitivity of the P-WFS. If the superposition of two Gaussian spots is considered, in fact, the measurement error to be associated to the tip-tilt determination is reduced for very small aberrations. Figure 4 shows that the reducing factor that needs to be applied is of the order of 10 for low values of $S$. This correction is neglected in the following. This is in fact an underestimation leading to a safer establishment of the predicted gain (i.e. the gain is likely to be lower bounded by the figures found here). However, numerical experiments show that the difference reported in Fig. 4 gets smaller for much lower $D / r_{0}$ and it is, moreover, confined to the solely tip-tilt term.

\subsection{Strehl ratio effect extended to higher orders}

Since in Eq. (3) the P-WFS variance is still proportional to the SH-WFS one, we can again introduce the argument of the Heisenberg uncertainty principle. Since the sensitivity of the sensor is inversely proportional to the second power of the focal spot linear dimension, we can extrapolate the dependence of the propagation coefficients for the other modes from the tip-tilt one as

$\sigma_{P-Z(q)}^{2} \approx\left[S \cdot\left(\frac{Q}{q}-1\right)+1\right]^{-2} \sigma_{\mathrm{SH}-Z(q)}^{2}$.

Figure 5 shows the error propagation coefficients for both the SH-WFS and the P-WFS in the noiseless case (black lines, the same data presented in Ragazzoni \& Farinato 1999), compared to the same errors for different SRs achieved on the pyramid pin in closed loop. The difference between the couple of curves representing the SH and the pyramid WFSs decreases as the performance in terms of SR tends to zero. This is expected since, for low SR values, the spot on the pyramid pin gets more similar to the one the system sees in open loop. 


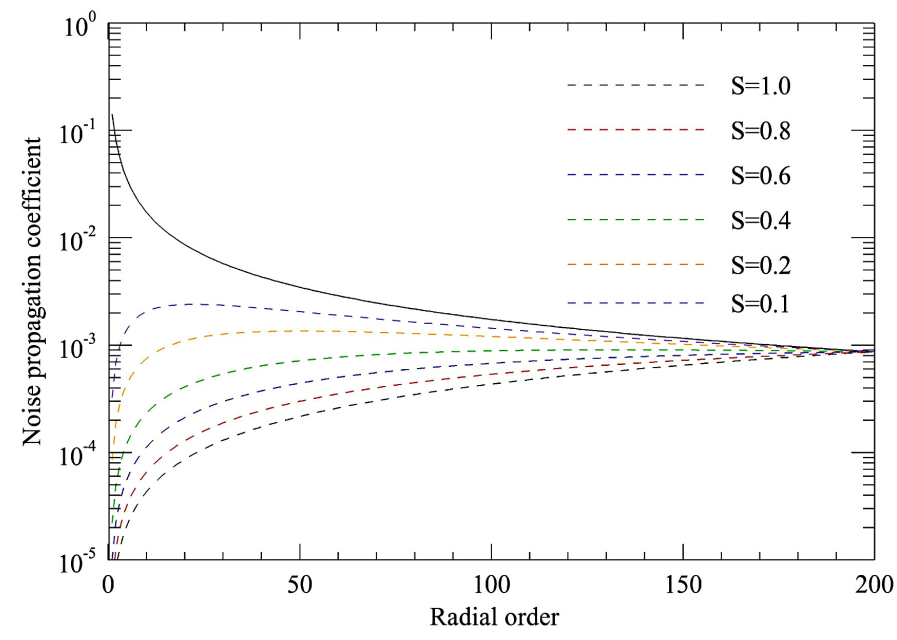

Fig. 5. Solid line: noise propagation coefficients vs. the radial order $q$ for the SH-WFS case; dashed lines: the same for the P-WFS case. Colors represent the effect of different SR achieved on the pyramid pin in closed loop. The $x$-axis maximum limit represents the $D / r_{0}$ parameter for the selected case (E-ELT-like telescope in $r_{0}=0.2 \mathrm{~m}$ conditions).

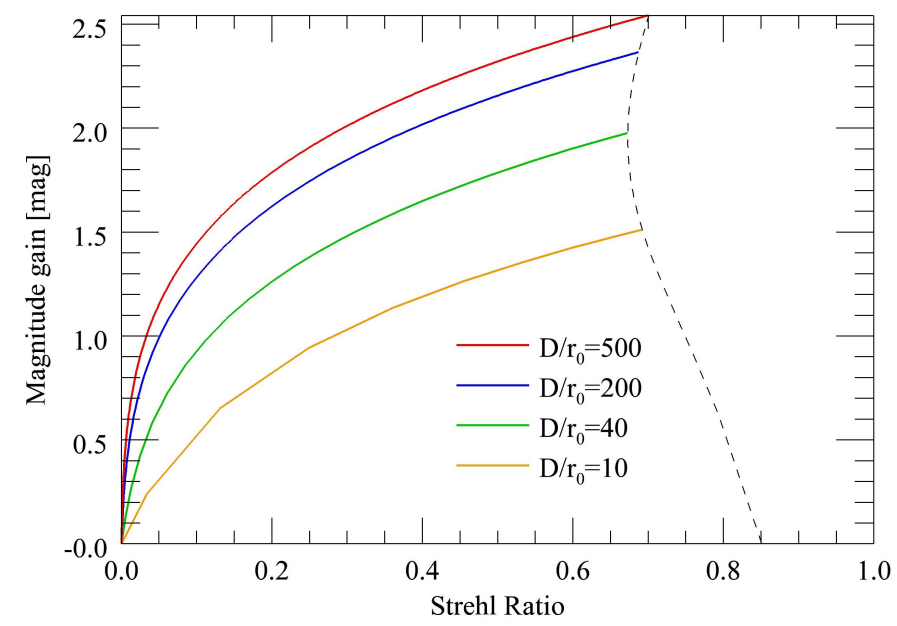

Fig. 6. Gain in limiting magnitude $\left(\Delta_{\mathrm{MAG}}\right)$ for different $Q=D / r_{0}$, as a function of the achieved SR on the pyramid pin. The dashed line shows the limit in the maximum SR that can be obtained for each $Q$-value.

The resulting $\Delta_{\mathrm{MAG}}$, which is now dependent on the SR, is shown in Fig. 6, for different values of $Q=D / r_{0}$. As we can see, there is a limit on the maximum achievable $S R$ value, which is related to the maximum corrected radial order. As per Noll (1976), in fact, the residual root mean square error, after the correction of the first $J$ (with $J>10$ ) Zernike modes of a wavefront perturbed according to Kolmogorov statistics, is

$\sigma_{J} \approx \sqrt{0.2944 J^{-\sqrt{3} / 2}\left(D / r_{0}\right)^{5 / 3}}[\mathrm{rad}]$,

whose corresponding limit SR is given by the Marechal's approximation as

$S_{J} \approx \exp \left(-0.2944 J^{-\sqrt{3} / 2}\left(D / r_{0}\right)^{5 / 3}\right)$.

In the realm of parameters considered in this work, the limit is close to $70 \%$.

For a wavefront sensor working in the visible band and installed at an ELT-like telescope, $D / r_{0}$ is usually above 200, which means that a relatively low SR, about $5 \%$, can still be large

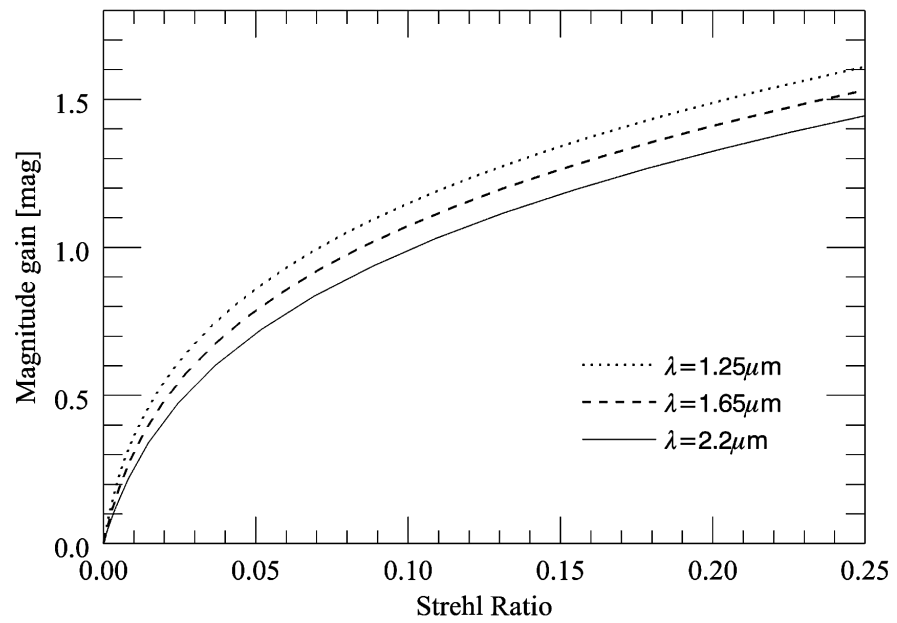

Fig. 7. Gain in limiting magnitude $\Delta_{\mathrm{MAG}}$ for different sensing wavelengths, for an E-ELT-like telescope (40 m diameter) with a standard ESO Paranal atmosphere.

enough to take advantage of the gain in sensitivity of a P-WFS over a SH-WFS, since a $\Delta_{\mathrm{MAG}} \sim 1 \mathrm{mag}$ is expected. For lower SR, however, the curves presented in Fig. 6 dramatically drop to $\Delta_{\mathrm{MAG}}=0$.

\section{Discussion: which is the gain for a low SR regime?}

As already mentioned, the P-WFS is not only used as a tool to reach very high resolution in classical $\mathrm{AO}$, but it can also be implemented in other $\mathrm{AO}$ techniques, such as $\mathrm{MCAO}$, ground layer AO (Rigaut 2002), multi-objects AO (Hammer et al. 2002), or in the recently proposed global MCAO (Ragazzoni et al. 2010). In all these cases, the P-WFS might operate in open or partially open loop and sense the turbulence at a shorter wavelength with respect to those for which the loop parameters are optimized, which leads to very low SRs on the pyramid pin. In this framework, it is important to evaluate which is the gain that can be expected for a system working at low SR by design. Figure 7 shows the expected $\Delta_{\mathrm{MAG}}$ for an E-ELT-like telescope, with a Fried parameter taken from the standard ESO Paranal atmosphere $\left(r_{0}=0.129 \mathrm{~m}\right.$ at $\left.500 \mathrm{~nm}\right)$, focusing on the low SR regime. The gain is here computed for different wavelengths, showing the loss in the $\Delta_{\mathrm{MAG}}$ value experienced by the system when moving to larger wavelengths. We note that, since a $r_{0}$ spatial sampling at the level of the pupil telescope is still assumed, for shorter wavelengths a higher sampling is automatically considered. Equation (6) has been used to derive SR.

If we consider the possibility of having a P-WFS working in the NIR regime, and we want to estimate what could be the actual $\Delta_{\text {MAG }}$ with respect to an analogous $\mathrm{SH}$ system, we can refer to the performances obtained by an existing P-WFS (FLAO), whose results also include error sources that we did not take into account in our model. Figure 8 shows the performance confirmed by FLAO at the LBT in $K$-band and an extrapolation to shorter wavelengths, which was performed assuming a constant corrected wavefront shape. We report this as having an estimation of the SR (which translates into gain in limiting magnitude) that we can expect from a real working system. If we focus on the faint-end regime (between 15 and $18.5 \mathrm{mag}$ ) and the $J$ band, we see that the SRs which can be achieved are always below $20 \%$. This, however, is a lower limit, since the performance here is 


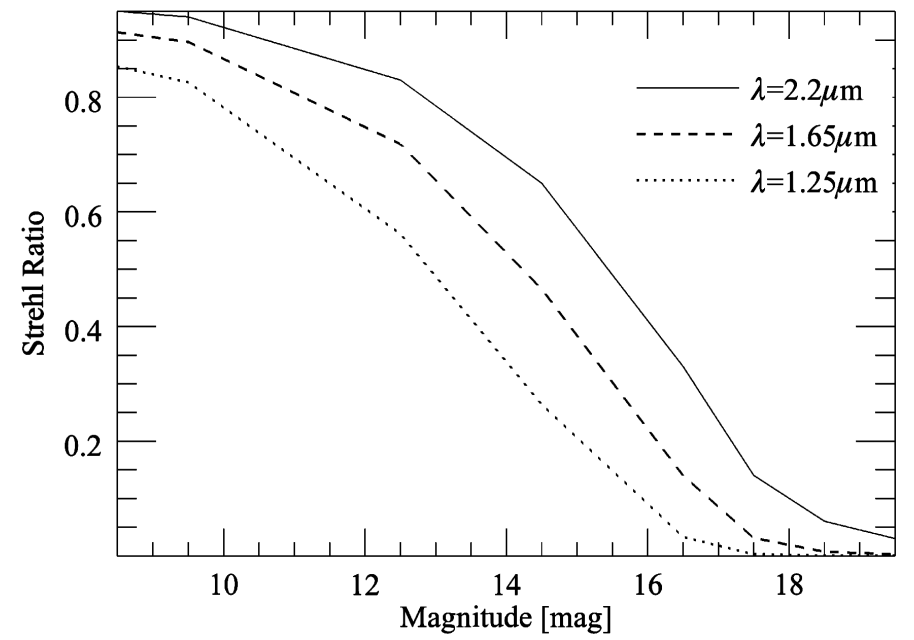

Fig. 8. FLAO performance in $K$ band (solid line), from Esposito et al. (2011), and the one extrapolated for a shorter wavelength.

measured on the scientific camera, and not directly on the pyramid pin. If we look at the curves in Fig. 7, with these types of SR and at NIR wavelengths, we can still expect a $\Delta_{\text {MAG }}$ value of up to $1.5 \mathrm{mag}$, which is a non-negligible increase.

\section{Discussion: dimensioning the system}

As just discussed, to take advantage of the gain in sensitivity of the P-WFS, a minimum SR on the pyramid pin itself should be reached. Another topic affected by this is the dimensioning of the system also in terms of hardware components, since the required pupil sampling is obviously the driver for the choice of both the detector and the correcting device (i.e. deformable mirror). In this section, we also explore the consequence of different spatial samplings at the level of the pupil on the gain parameter. The effect of different samplings on the SR or the $\Delta_{\mathrm{MAG}}$ is of course related to the considered wavelength, since the Fried parameter directly depends on it. Figure 9 shows the computed $\Delta_{\text {MAG }}$ with limited correction in terms of SR on the pyramid pin, as a function of the maximum corrected radial order, for an E-ELT-like system (a 40 m diameter pupil was considered). The expected SR is directly derived from the pupil sampling under noiseless assumption from high spatial frequency residuals (Noll 1976), and the result is shown for different corrected radial orders and the full considered wavelength range, in Fig. 10. The plot shows the result of our simulation for a set of different wavelengths, with a $0.1 \mu \mathrm{m}$ separation between each of them in the $0.5-2.2 \mu \mathrm{m}$ range. With this result, we can find a relation between the pupil sampling in $r_{0}$ units (thus including the wavelength dependence), to expect a certain gain $\Delta_{\mathrm{MAG}}$. Even if the precise values depend on the approximation used to estimate the number of actuators to correct a given radial order, we can derive a rule of thumb. In fact, to experience any $\Delta_{\mathrm{MAG}}$, one should tune the $\mathrm{AO}$ correction parameters to spatially sample the pupil with a pitch lower than $5-6 r_{0}$. If we want to take advantage of a 1 mag gain, instead, the pitch should be smaller than $\sim 3 r_{0}$, while a pitch $\sim r_{0}$ is required to aim for a gain of 2 mag.

\section{Conclusions}

Defining $\Delta_{\text {MAG }}$ as the non-modulated P-WFS gain in the reference star limiting magnitude with respect to an SH-WFS, in perfect closed loop conditions and with the same sampling, we

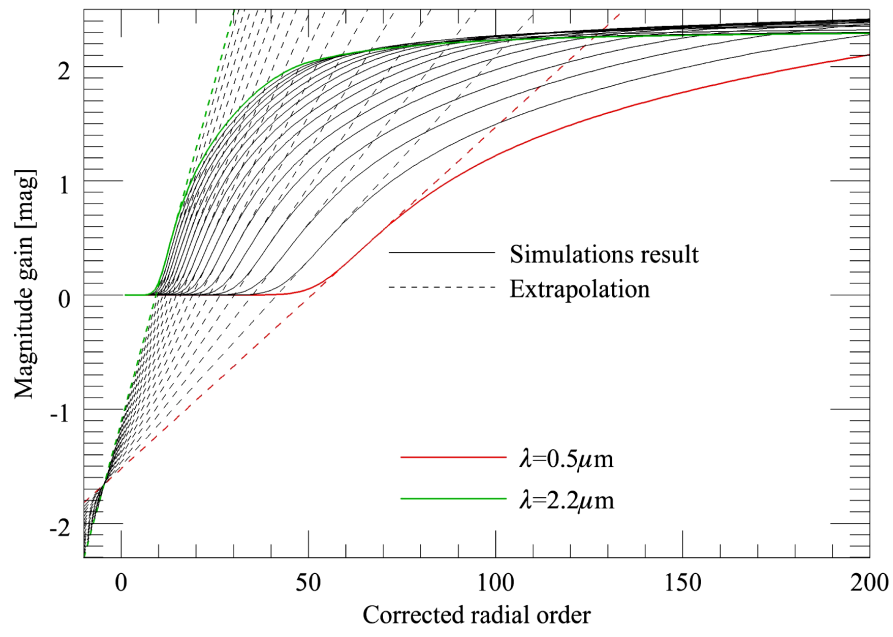

Fig. 9. Gain in magnitude of a P-WFS with respect to an SH-WFS $\left(\Delta_{\mathrm{MAG}}\right)$ in a limited SR regime, as a function of the maximum radial order that can be corrected by the deformable mirror. Solid lines represent the computed gain for different wavelengths, with a $0.1 \mu \mathrm{m}$ separation between each of them. The extreme wavelengths considered are displayed in green $(\lambda=2.2 \mu \mathrm{m})$ and red $(\lambda=0.5 \mu \mathrm{m})$, being the correspondent $D / r_{0}$ parameters 52 and 310 , respectively. Dashed lines represent the maximum slopes of the curves.

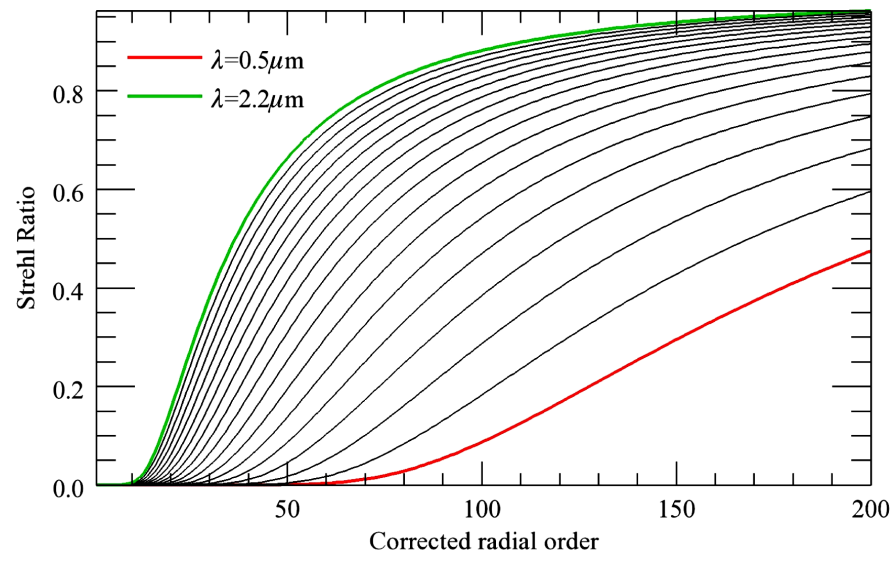

Fig. 10. SR as a function of the corrected radial order for different wavelengths. This is directly derived from the pupil sampling under noiseless assumption from high spatial frequency residuals. The extreme wavelengths considered are displayed in green $(\lambda=2.2 \mu \mathrm{m})$ and red $(\lambda=0.5 \mu \mathrm{m})$.

retrieved the dependence of $\Delta_{\mathrm{MAG}}$ on the SR that was achieved on the pyramid pin. Since the gain is due to the fact that the reference star image at the entrance of the P-WFS shrinks, partial wavefront correction conditions reduce the $\Delta_{\text {MAG }}$ value with respect to the one expected for a noiseless ideal system. Assuming a spatial sampling down to the Fried parameter, we retrieved $\Delta_{\text {MAG }}$ as a function of the SR for different $D / r_{0}$, obtaining the result that, for an ELT-class telescope in the Visible band, a $\Delta_{\mathrm{MAG}} \sim 1 \mathrm{mag}$ is expected for a $5 \% \mathrm{SR}$, while a higher SR of about $40 \%$ translates into a gain $\Delta_{\mathrm{MAG}} \sim 2 \mathrm{mag}$. Focusing on the low SR regime, we found that the gain can be described as a function of the spatial sampling in terms of the Fried parameter. As a rule of thumb, we can say that, to obtain a $\Delta_{\text {MAG }}$ higher than $0 \mathrm{mag}, 1 \mathrm{mag}$, and $2 \mathrm{mag}$, the spatial sampling of the pupil needs to be denser than $5-6 r_{0}, \sim 3 r_{0}$, and $\sim r_{0}$, respectively. This result can be taken into account for the selection of system parameters, since this low SR regime is in the realm of most of the 
wide field correction AO systems. Moreover, it can also apply to the cases in which the WFS is working at a shorter wavelength with respect to the scientific camera, for which the pupil spatial sampling is dimensioned.

Acknowledgements. The authors greatly appreciated the useful review of the anonymous referee.

\section{References}

Arcidiacono, C., Diolaiti, E., Tordi, M., et al. 2004, Appl. Opt., 43, 4288

Beckers, J. M. 1988, in Very Large Telescopes and their Instrumentation, ESO Conf., 2, 693

Chamot, S. R., Dainty, C., \& Esposito, S. 2006, Opt. Exp., 14, 518

Esposito, S., \& Riccardi, A. 2001, A\&A, 369, L9

Esposito, S., Riccardi, A., Pinna, E., et al. 2011, in SPIE Conf. Ser., 8149, 2

Foy, R., \& Labeyrie, A. 1985, A\&A, 152, L29

Ghedina, A., Cecconi, M., Ragazzoni, R., et al. 2003, in Adaptive Optical System Technologies II, eds. P. L. Wizinowich, \& D. Bonaccini, SPIE Conf. Ser., 4839,869

Gilmozzi, R., \& Spyromilio, J. 2007, The Messenger, 127, 11

Guyon, O. 2010, PASP, 122, 49

Hammer, F., Sayède, F., Gendron, E., et al. 2002, in Scientific Drivers for ESO Future VLT/VLTI Instrumentation, eds. J. Bergeron, \& G. Monnet, 139

Hardy, J. W., Lefebvre, J. E., \& Koliopoulos, C. L. 1977, J. Opt. Soc. Am., 67, 360

Herbst, T., Ragazzoni, R., Andersen, D., et al. 2003, in Interferometry for Optical Astronomy II, ed. W. A. Traub, SPIE Conf. Ser., 4838, 456

Hill, J. M., \& Salinari, P. 2000, in Telescope Structures, Enclosures, Controls, Assembly/Integration/Validation, and Commissioning, eds. T. A. Sebring, \& T. Andersen, SPIE Conf. Ser., 4004, 36
Iglesias, I. 2011, Opt. Lett., 36, 3636

Iglesias, I., Ragazzoni, R., Julien, Y., \& Artal, P. 2002, Opt. Exp., 10, 419 Johns, M. 2008, in SPIE Conf. Ser., 6986, 3

Kasper, M., Verinaud, C., \& Beuzit, J.-L. 2014, in Search for Life Beyond the Solar System. Exoplanets, Biosignatures \& Instruments, eds. D. Apai, \& P. Gabor, 4

Le Louarn, M., Foy, R., Hubin, N., \& Tallon, M. 1998, MNRAS, 295, 756

Marchetti, E., Brast, R., Delabre, B., et al. 2007, The Messenger, 129, 8

Noll, R. J. 1976, J. Opt. Soc. Am., 66, 207

Peter, D., Feldt, M., Henning, T., et al. 2010, PASP, 122, 63

Ragazzoni, R. 1996, J. Mod. Opt., 43, 289

Ragazzoni, R., \& Farinato, J. 1999, A\&A, 350, L23

Ragazzoni, R., Baruffolo, A., Farinato, J., et al. 2000a, in Adaptive Optical Systems Technology, ed. P. L. Wizinowich, SPIE Conf. Ser., 4007, 57

Ragazzoni, R., Farinato, J., \& Marchetti, E. 2000b, in Adaptive Optical Systems Technology, ed. P. L. Wizinowich, SPIE Conf. Ser., 4007, 1076

Ragazzoni, R., Diolaiti, E., Farinato, J., et al. 2002, A\&A, 396, 731

Ragazzoni, R., Arcidiacono, C., Dima, M., et al. 2010, in Adaptative Optics for Extremely Large Telescopes, Conf. (EDP Sciences), 02003

Ribak, E. N., \& Rigaut, F. 1994, A\&A, 289, L47

Rigaut, F. 2002, in European Southern Observatory Conf. and Workshop Proc., eds. E. Vernet, R. Ragazzoni, S. Esposito, \& N. Hubin, 58, 11

Rigaut, F., \& Gendron, E. 1992, A\&A, 261, 677

Roddier, F. 1981, Prog. Opt. (Amsterdam: North-Holland Publishing Co.), 19, 281

Roddier, F., \& Roddier, C. 1988, in Very Large Telescopes and their Instrumentation, ESO Conf., 2, 667

Smartt, R. N., \& Strong, J. 1974, Opt. Eng., 13, G198

Szeto, K., Roberts, S., Gedig, M., et al. 2008, in SPIE Conf. Ser., 7012, 2

Verinaud, C. 2004, Opt. Comm., 233, 27

Weiner, J., Danchi, W. C., Hale, D. D. S., et al. 2000, ApJ, 544, 1097 